\title{
An expedient general synthesis of pyrrolo[3,2-e]indazoles: domino Sonogashira/Cacchi coupling-heteroannulation reactions
}

\author{
Sujoy K. Barik, ${ }^{a}$ Moumita Rakshit, ${ }^{b}$ Gandhi K. Kar, ${ }^{b}$ and Manas Chakrabarty* ${ }^{a}$ \\ ${ }^{a}$ Department of Chemistry, Bose Institute, 93/1 A.P.C. Road, Kolkata-700 009, India \\ ${ }^{b}$ Department of Chemistry, Presidency University, 86/1 College Street, Kolkata-700 073, India \\ E-mail: chakmanas09@gmail.com
}

DOI: $\underline{\text { http://dx.doi.org/10.3998/ark.5550190.p008.596 }}$

\begin{abstract}
A one-pot Sonogashira coupling-heteroannulation of 4-iodo-1-phenylsulfonyl-5-trifluoroacetamidoindazole with terminal acetylenes using bis(triphenylphosphine)palladium(II) dichloride as the catalyst, cuprous iodide as the co-catalyst and triethylamine as the base in DMF furnished, after N(3)-deprotection, 7-H/substituted 3,6-dihydropyrrolo[3,2-e]indazoles in high yields. This is the first general synthesis of pyrrolo[3,2-e]indazoles. Uncatalyzed hydrodesilylation was observed during reaction with trimethylsilylacetylene, leading to the 7 unsubstituted parent pyrrolo[3,2-e]indazole.
\end{abstract}

Keywords: Pyrrolo[3,2-e]indazoles, Sonogashira coupling/5-endo-dig cyclisation, palladium(II) catalyst, domino reactions

\section{Introduction}

The indole ring constitutes the structural core of a host of bioactive compounds of natural and synthetic origins. ${ }^{1,2}$ More than 10,000 bioactive indoles are known, of which over 200 are either used as drugs or are in clinical trials. ${ }^{3}$ Substituted indazoles too are of considerable pharmaceutical importance because of their therapeutic potential and use, ${ }^{4}$ amongst which benzydamine is a notable anti-inflammatory agent. ${ }^{5,6}$ In continuation of our ongoing interest in the development of general synthetic routes to potentially bioactive condensed nitrogen heterocycles, ${ }^{7-14}$ we recently focused our attention on a relatively less studied class of heterocycles which incorporate both the indole and the indazole nuclei, viz. pyrazoloindoles which may also be regarded as pyrroloindazoles. A report on the study of indazole-containing polycyclic compounds in search of antitumor agents ${ }^{15}$ strengthened our motivation.

The extant literature on the synthesis and bioactivity of pyrroloindazoles revealed that eight isomeric classes of pyrroloindazoles have so far been synthesised. ${ }^{16-33}$ Of these, only the $[2,3-g]$ - 
isomers $^{18-27}$ and the $[3,2-e]$-isomers ${ }^{22,24,25}$ display various biological activities. Thus, the [2,3-g]isomers 1-4 and the [3,2-e]-pyrroloindazole 5 were bioactive. While 1 showed analgesic and antiinflammatory activity, ${ }^{18} \mathbf{2}$ showed NMDA ( $N$-methyl D-aspartate)-receptor antagonistic property with potential for the treatment of neuropathic pain, ${ }^{22} \mathbf{3}$ and $\mathbf{4}$ displayed inhibition of sGC (soluble guanylate cyclase) with potential for treating hypertension ${ }^{23}$ whereas $\mathbf{5}$ proved to be an antagonist of NMDA-receptor. ${ }^{22}$

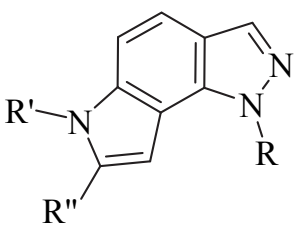

1. $\mathrm{R}=$ alkyl; $\mathrm{R}^{\prime}=\mathrm{H} ; \mathrm{R}^{\prime \prime}=\mathrm{Me}$

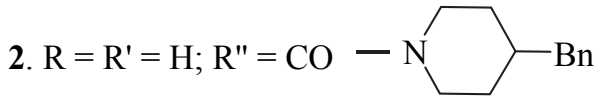

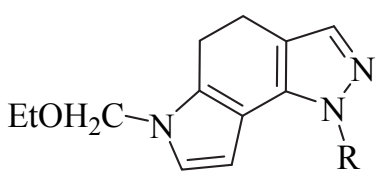

3. $\mathrm{R}=\mathrm{Me}$

4. $\mathrm{R}=\mathrm{Ph}$

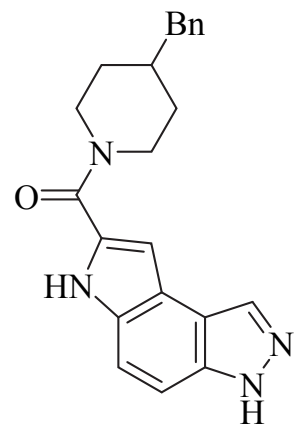

5

Among these two classes of pyrroloindazoles, only the [2,3-g]-types have already been subjected to rather thorough synthetic investigations. ${ }^{18-27}$ But the $[3,2-e]$-types were synthesized only twice, neither of which was a general route.

In the first synthesis of pyrrolo[3,2-e]indazoles, only one member, viz. the 5-carbethoxy derivative was prepared from 5-aminoindazole using Fischer/Japp-Klingemann procedure. The derived 4-benzylpiperidinylcarboxamide proved to be a good antagonist of NMDA-receptor. ${ }^{22}$ In the second synthesis, ${ }^{24,25}$ two substrates, viz. 6-H- and 6-bromo-4,5-indolynes, generated in situ, were separately treated with ethyl diazoacetate when a mixture of two isomeric pyrroloindazoles, viz. the [3,2-e]-type and the [2,3-g]-type were formed in each case. Thus, 4-H-1carbethoxypyrrolo[3,2-e]indazole and 8-H-1-carbethoxypyrrolo[2,3-g]indazole were formed from the 6-H-indolyne, whereas 4-bromo-1-carbethoxypyrrolo[3,2-e]indazole and 8-bromo-1carbethoxypyrrolo[2,3-g]indazole were formed from the 6-bromoindolyne.

The reported bioactivity, hence the bioactive potential of pyrrolo[3,2-e]indazoles and particularly the absence of a general synthetic route to this particular isomeric class called for the development of a general synthesis of pyrrolo[3,2-e]indazoles, which we have now accomplished and is reported herein.

Several classical routes to the synthesis of indoles are known. ${ }^{34,35}$ Indazoles too have been the subject of synthetic efforts during the past decades. ${ }^{36}$ Our plan was to utilize one of the recently developed palladium-catalyzed synthetic routes to indoles ${ }^{37,38}$ because of the mild reaction conditions involved, tolerance of a wide variety of functional groups (thereby avoiding the use of protecting groups), high regioselectivities and high yields.

The aminopalladation/reductive elimination domino reaction of alkynes containing a proximate nitrogen nucleophile, first observed by Yamanaka and subsequently by Cacchi, ${ }^{39}$ has 
proved to be very useful for the formation of a pyrrole ring incorporated into an indole nucleus. To be precise, Cacchi's novel approach to 2,3-disubstituted indoles through a palladiumcatalyzed cyclisation of ortho-alkynyl-trifluoroacetanilides with aryl, heteroaryl and alkenyl halides, specially iodides, or triflates ${ }^{40,41}$ was of interest to us. Later, Flynn modified conditions to develop a one-pot, two-step synthesis of only one 2,3-disubstituted indole using bis(triphenylphosphine)palladium(II) dichloride as the catalyst. ${ }^{42}$

\section{Results and Discussion}

Our plan was to utilize the Sonogashira-Hagihara protocol (more often simply called Sonogashira reaction) $)^{43-46} /$ Cacchi procedure for the construction of the pyrrole ring on the indazole framework. Our retrosynthetic analysis of the envisaged title molecules, shown in Scheme 1, involved 5-amino-N(1)-phenylsulfonylindazole ${ }^{11}$ as the starting material and it was prepared from 5-nitroindazole 6.

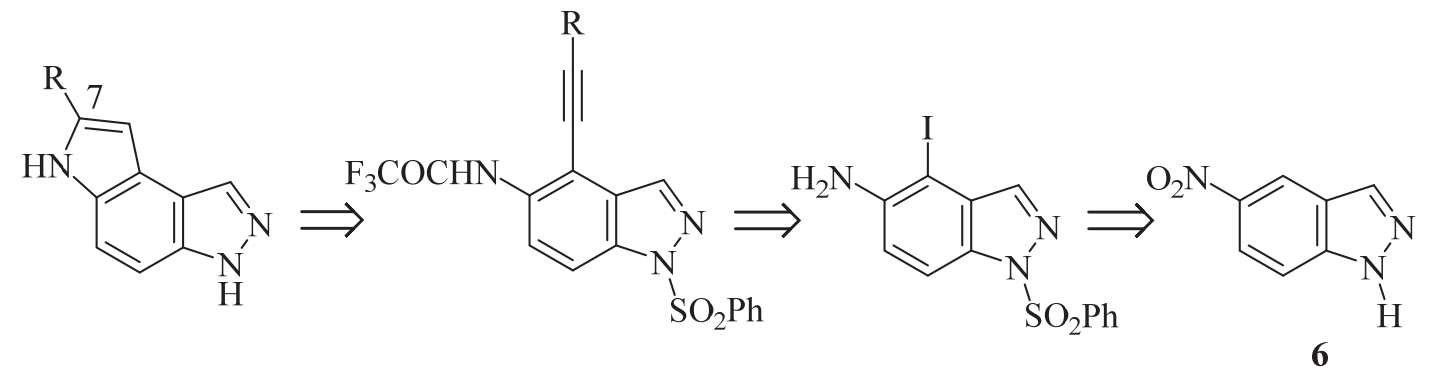

Scheme 1. Retrosynthesis of 7-substituted pyrrolo[3,2-e]indazoles.

Accordingly, the desired starting amine 8 was prepared from commercially available indazole 6 by successive N(1)-phenylsulfonylation (PTC-mediated) and reduction (hydrazine hydrate and palladized charcoal) of the resulting protected nitroindazole 7. It was then iodinated exclusively at C-4 by $N$-iodosuccinimide (NIS) to efficiently furnish $\mathbf{9}$. The site of iodination received support from the appearance of ${ }^{1} \mathrm{H}$ NMR signals at $\delta 7.09$ and ca. $7.84 \mathrm{ppm}(1 \mathrm{H}$, d each $J 9 \mathrm{~Hz}$ ) in the ${ }^{1} \mathrm{H}$ NMR spectrum of 9, which corresponded to H-6 and H-7. The aminoiodoindazole 9 was trifluoroacetylated to furnish the Sonogashira/Cacchi substrate, ortho-iodotrifluoroacetamidoindazole $\mathbf{1 0}$ in very good yield (Scheme 2). 


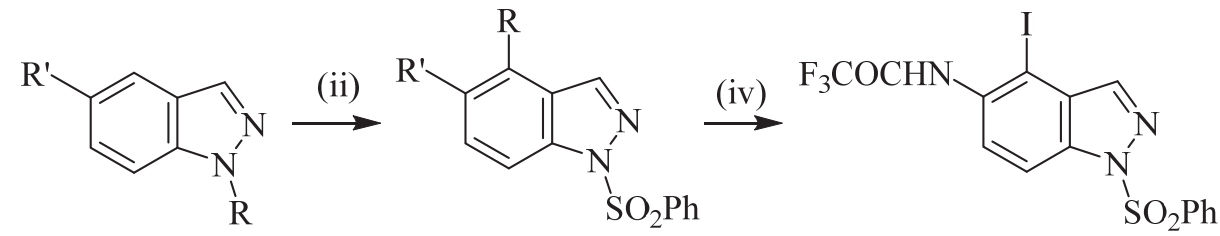

(i)
6: $\mathrm{R}=\mathrm{H} ; \mathrm{R}^{\prime}=\mathrm{NO}_{2}$
$\rightarrow 7: \mathrm{R}=\mathrm{SO}_{2} \mathrm{Ph} ; \mathrm{R}^{\prime}=\mathrm{NO}_{2}$
(iii) 8: $\mathrm{R}=\mathrm{H} ; \mathrm{R}^{\prime}=\mathrm{NH}_{2}$
9: $\mathrm{R}=\mathrm{I} ; \mathrm{R}^{\prime}=\mathrm{NH}_{2}$

10

Reaction conditions and yields: (i) $\mathrm{PhSO}_{2} \mathrm{Cl}, \mathrm{NaOH}, n-\mathrm{Bu}_{4} \mathrm{~N}^{+} \mathrm{HSO}_{4}^{-}$, THF, rt, 1 h; (7): $84 \%$; (ii) $\mathrm{NH}_{2} \mathrm{NH}_{2}$, $\mathrm{Pd} / \mathrm{C}, \mathrm{MeOH}$, reflux, 3 h; (8): 82\%; (iii) NIS, $\mathrm{CH}_{3} \mathrm{CN}$, rt, 2 h; (9): 81\%; (iv) TFAA, $\mathrm{Et}_{3} \mathrm{~N}, \mathrm{THF}, 0{ }^{\circ} \mathrm{C}, 2 \mathrm{~h}$; (10): $83 \%$.

Scheme 2. Preparation of the key precursor 10.

The reaction of the Sonogashira/Cacchi substrate 10 was first tried with TMSA 11a since TMSAs can be hydrodesilylated using a fluoride source, mostly TBAF, or an inorganic base, usually aq. KOH-MeOH. We used bis(triphenylphosphine)palladium(II) dichloride $\left[\mathrm{Pd}\left(\mathrm{Ph}_{3} \mathrm{P}\right)_{2} \mathrm{Cl}_{2}\right](10 \mathrm{~mol} \%)$ as the catalyst in our experiments. Thus, 10 was treated with $11 \mathrm{a}(1.4$ equiv) and $\mathrm{Pd}\left(\mathrm{Ph}_{3} \mathrm{P}\right)_{2} \mathrm{Cl}_{2}(10 \mathrm{~mol} \%)$ in $\mathrm{DMF}$ in the presence cuprous iodide $(10 \mathrm{~mol} \%)$ as the co-catalyst and $\mathrm{Et}_{3} \mathrm{~N}$ (10 equiv) as the base and stirred at $100-110^{\circ} \mathrm{C}$ in an argon atmosphere (to avoid Hay-Glaser homocoupling of terminal acetylenes ${ }^{47}$ ) until the substrate was consumed. The optimal concentrations of the catalyst, the co-catalyst and the base were determined by trial experiments which have not been described in the Experimental Section. A usual work-up of the reaction mixture, followed by purification of the crude product by column chromatography over silica gel, furnished a single product. Though expected to be the corresponding 7-TMSpyrroloindazole, the sole product was indeed identified as 7-unsubstituted 3-phenylsulfonyl-3,6dihydropyrrolo[3,2-e]indazole 12a by combined spectroscopic (IR, ${ }^{1} \mathrm{H}$ and ${ }^{13} \mathrm{C} \mathrm{NMR}, \mathrm{MS}$ ) and elemental analyses. The presence of two ${ }^{1} \mathrm{H}$ NMR signals at $\delta 7.71$ and $7.87 \mathrm{ppm}(1 \mathrm{H}$, each d, $J$ $9 \mathrm{~Hz}$ ), corresponding to $\mathrm{H}-4$ and $\mathrm{H}-5$ of the pyrrolo[3,2-e]indazole nucleus, lent support to its type of isomer. It thus transpired that the tandem Sonogashira/Cacchi coupling-heteroannulation as well as hydrodesilylation had occurred in one pot.

The observed cleavage of the TMS group occurring under the tandem SonogashiraHagihara/Cacchi cross-coupling-heteroannulation reactions requires explanation since we did not use any hydrodesilylating agent in our experiment. We believe, direct palladium-coppercatalyzed coupling of $\mathbf{1 0}$ with $\mathbf{1 1 a}$ in a so-called "sila"-Sonogashira reaction" led to the elimination of trimethylsilyl iodide with the formation of ortho-ethynyl-trifluoroacetamidoindazole. It was immediately followed by 5-endo-dig cyclisation to form $\mathbf{1 2 a}$.

The occurrence of two reactions in tandem and the high yield of the product encouraged us to check the generality of the reaction. Hence, $\mathbf{1 0}$ was separately treated with seven other terminal acetylenes 11b-h under the aforesaid conditions. Gratifyingly, the respective 7-substituted 3phenylsulfonyl-3,6-dihydropyrrolo[3,2-e]indazoles $\mathbf{1 2 b}$-h were isolated as the sole products in 
$82-94 \%$ yields in 5-8 $\mathrm{h}$ (Table 1). In the ${ }^{1} \mathrm{H}$ NMR spectra of all these products, the signals for $\mathrm{H}-$ 4 and $\mathrm{H}-5$ appeared as $1 \mathrm{H}$, doublet each in the range $\delta 7.59-8.0 \mathrm{ppm}$ showing, as expected, an ortho-coupling $(J 8.5-9.5 \mathrm{~Hz})$. The formation of [3,2-e]-type of pyrroloindazoles was thus confirmed. Pertinently, H-1 appeared at $\delta 8.6-8.9$ ppm (s) in 12a-h, whereas H-8 appeared at $c a$. $\delta 6.5 \mathrm{ppm}$ (s) in the 7-alkyl derivatives $\mathbf{1 2 b}$-e and at $\delta 7.2-7.3 \mathrm{ppm}(\mathrm{s})$ in the 7-aryl derivatives 12f-h.

Table 1. Synthesis of 7-H/substituted 3,6-dihydropyrrolo[3,2-e]indazoles 12a-h from terminal acetylenes using domino reactions

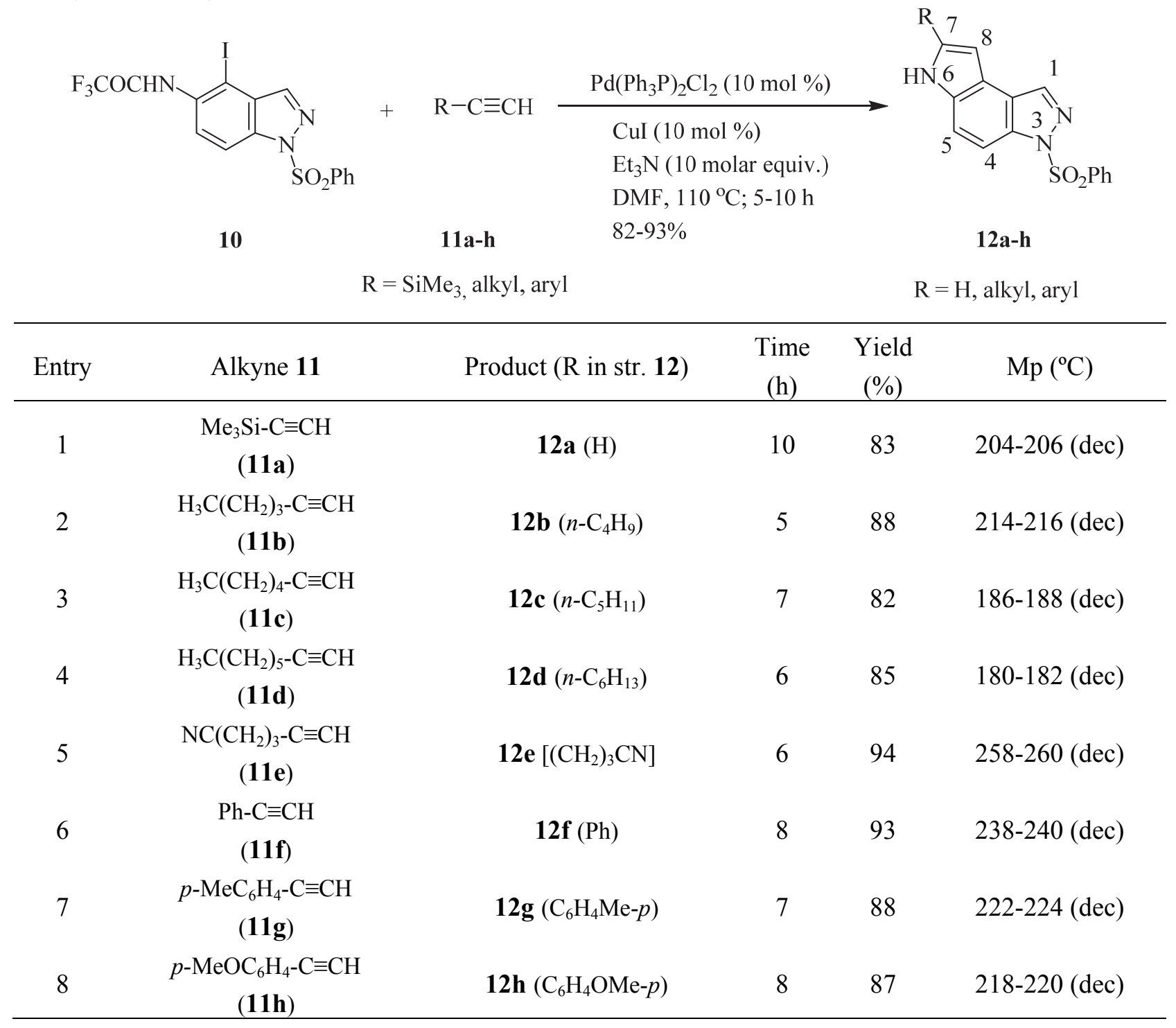

For $N(3)$-deprotection, each of 12a-h was refluxed with aqueous methanolic potassium carbonate for $2 \mathrm{~h}$, which generated the parent $7 \mathrm{H} /$ substituted 3,6-dihydropyrrolo[3,2-e]indazoles (13a-h) in 85-91\% yields (Table 2). As expected, H-1 showed an upfield shift (to $\delta$ 8.1-8.3 ppm, s) in 13a-h, whereas there was not much effect on the chemical shifts of H-8. 
Table 2. Synthesis of 7-substituted 3,6-dihydropyrrolo[3,2-e]indazoles via $\mathrm{N}$-deprotection of $N(3)$-phenylsulfonylpyrroloindazoles<smiles>[R]c1cc2c(ccc3c2cnn3S(=O)(=O)O)[nH]1</smiles>

12a-h

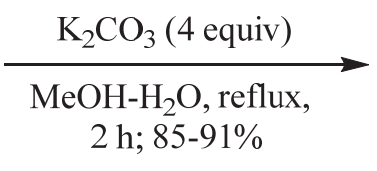

$\mathrm{R}=\mathrm{H}$, alkyl, aryl<smiles>[R]c1cc2c(ccc3[nH]nc(I)c32)[nH]1</smiles>

13a-h

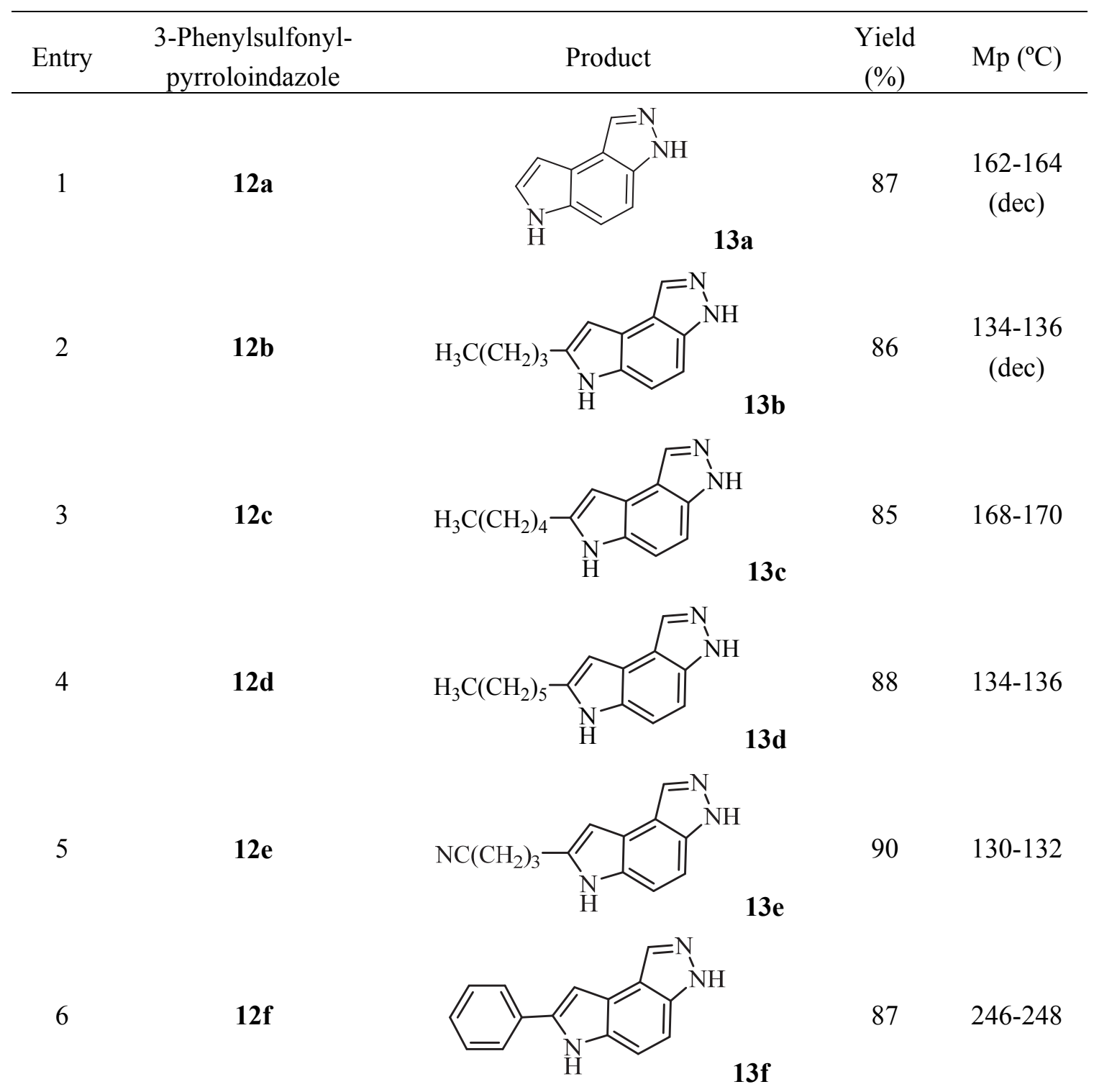


Table 2 (continued)

7

8
$12 \mathrm{~g}$<smiles>Cc1ccc(-c2cc3c(ccc4[nH]ncc43)[nH]2)cc1</smiles>

12h

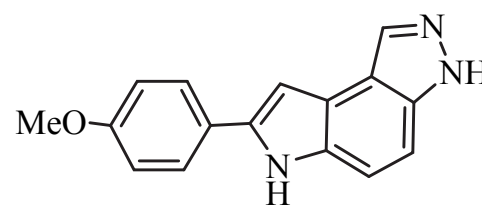

91

$>250$

$13 g$

86 $>250$

\section{Conclusions}

An efficient, general synthesis of 7-H/substituted 3,6-dihydropyrrolo[3,2-e]indazoles has been developed by the reaction of 4-iodo-1-phenylsulphonyl-5-trifluoroacetamidoindazole with terminal alkynes using Sonogashira/Cacchi coupling-heteroannulation reactions, followed by $N(3)$-deprotection. To the best of our knowledge, this is the first general synthesis of this class of compounds, that too involving domino reactions. The overall procedure is simple, the yields are consistently high and both the indolic and the indazolic nitrogens are amenable to derivatization, thereby rendering our method even more useful. Furthermore, our work opens up the study of the reactions of $\mathbf{1 0}$ with internal alkynes, which is likely to unveil interesting results on 7,8disubstituted pyrroloindazoles.

Pertinently, after the completion of our work, a report was published only a few months ago on a convenient synthesis of pyrrolo[3,4-g]indazoles by the annelation of the pyrazole ring on the isoindole moiety, and four members of the synthesized pyrroloindazoles showed modest antitumor activity against a host of human tumor cell lines. ${ }^{49}$

\section{Experimental Section}

General. All melting points (in Celsius) were recorded on a Toshniwal apparatus. The IR spectra were recorded on $\mathrm{KBr}$ pellets using a Perkin-Elmer-782 spectrophotometer, the ${ }^{1} \mathrm{H}(500 \mathrm{MHz})$ and ${ }^{13} \mathrm{C}(125 \mathrm{MHz})$ NMR spectra (PND, DEPT 135) (except in one case) on a Bruker DRX 500 NMR spectrometer unless otherwise mentioned, the LR EI-MS on a JEOL JMSAX505HA and HR ESI-MS(+) on a Waters Qtof Micro YA263 mass spectrometer. All elemental analyses were carried out on Perkin Elmer 2400 Series II C, H, N Analyzer. Analytical TLCs were carried out on silica gel G (Merck, India) plates. PE refers to petroleum ether, bp $60-80{ }^{\circ} \mathrm{C}$. Column chromatography (CC) was performed using silica gel (100-200 mesh). DMF was dried, distilled 
and stored over molecular sieves (4 $\AA$ ). 5-Nitroindazole, $\mathrm{Pd} / \mathrm{C}$ and all terminal alkynes were procured from Sigma-Aldrich. Phenylsulfonyl chloride, trifluoroacetic anhydride and hydrazine hydrate were purchased from Spectrochem, India. $N$-Iodosuccinimide (NIS) was purchased from Alfa-Aesar.

Synthesis of 5-amino-4-iodo-1-phenylsulfonylindazole (9). To a solution of 5-amino-1phenylsulfonylindazole $(8)^{11}(2.73 \mathrm{~g}, 10 \mathrm{mmol})$ in dry $\mathrm{MeCN}(60 \mathrm{~mL})$ at $\mathrm{rt}$ was gradually added NIS $(2.70 \mathrm{~g}, 12 \mathrm{mmol})$ and the solution stirred until $(2 \mathrm{~h})$ the reaction was complete. The solution was poured into $10 \%$ aqueous $\mathrm{Na}_{2} \mathrm{~S}_{2} \mathrm{O}_{3}$ and $\mathrm{MeCN}$ distilled off. The solution was extracted with EtOAc $(3 \times 50 \mathrm{~mL})$, the organic layer separated and washed with $\mathrm{H}_{2} \mathrm{O}$, dried $\left(\mathrm{Na}_{2} \mathrm{SO}_{4}\right)$ and the solvent removed under vacuum. The resulting dark colored residue was purified by CC (10\% EtOAc in PE) to furnish 9 as a colorless solid, yield $81 \%, 3.23 \mathrm{~g}, \mathrm{mp} 182-184{ }^{\circ} \mathrm{C}$; IR ( $v_{\max }, \mathrm{cm}^{-}$ $\left.{ }^{1}\right): 3468,3375,1614,1505,1366,1176,1058,817,725 .{ }^{1} \mathrm{H} \mathrm{NMR}\left(\mathrm{CDCl}_{3}\right): \delta_{\mathrm{H}} 5.46\left(2 \mathrm{H}, \mathrm{s}, \mathrm{NH}_{2}\right)$, $7.09(1 \mathrm{H}, \mathrm{d}, J 9 \mathrm{~Hz}), 7.51\left(2 \mathrm{H}, \mathrm{td}, J_{1} 7.5 \mathrm{~Hz}, J_{2} 1.5 \mathrm{~Hz}\right), 7.67\left(1 \mathrm{H}, \mathrm{tt}, J_{1} 7.5 \mathrm{~Hz}, J_{2} 1 \mathrm{~Hz}\right), 7.84$ $(1 \mathrm{H}, \mathrm{d}, J 9 \mathrm{~Hz}), 7.82-7.85(2 \mathrm{H}, \mathrm{m}), 8.06(1 \mathrm{H}, \mathrm{d}, J 1 \mathrm{~Hz}) .{ }^{13} \mathrm{C} \mathrm{NMR}\left(\mathrm{CDCl}_{3}\right): \delta_{\mathrm{C}} 113.8,119.3$, 127.4, 130.2, 135.3, 144.7 (all $\mathrm{CH}_{\text {arom}}$ ), 69.8, 131.4, 133.3, 136.6, 147.2 (all $\mathrm{C}_{\text {arom}}$ ). MS (EI), $m / z$ $(\%)=399(100)\left(\mathrm{M}^{+}\right), 258(45)\left(\mathrm{M}-\mathrm{SO}_{2} \mathrm{Ph}\right)$. Anal. Calcd for $\mathrm{C}_{13} \mathrm{H}_{10} \mathrm{IN}_{3} \mathrm{O}_{2} \mathrm{~S}: \mathrm{C}, 39.10 ; \mathrm{H}, 2.51 ; \mathrm{N}$, $10.53 \%$. Found: C, 39.15; H, 2.50; N, 10.56\%.

Synthesis of 4-iodo-1-phenylsulfonyl-5-(trifluoroacetamido)indazole (10). To a solution of 9 (3.98 g, $10 \mathrm{mmol})$ in dry THF $(75 \mathrm{~mL})$ was added $\mathrm{Et}_{3} \mathrm{~N}(2 \mathrm{~mL})$ and $\left(\mathrm{CF}_{3} \mathrm{CO}\right)_{2} \mathrm{O}(1.95 \mathrm{~mL})$ at 0 ${ }^{\circ} \mathrm{C}$, and the reaction mixture was stirred until $(2 \mathrm{~h})$ the reaction was complete. The solution was diluted with $\mathrm{H}_{2} \mathrm{O}(40 \mathrm{~mL})$, THF distilled off and the aqueous phase extracted with EtOAc $(3 \times 50$ $\mathrm{mL})$. The combined solvent extracts were washed with $\mathrm{H}_{2} \mathrm{O}$, dried $\left(\mathrm{Na}_{2} \mathrm{SO}_{4}\right)$ and the solvent removed to furnish a crude product which was purified by $\mathrm{CC}(10 \% \mathrm{EtOAc} / \mathrm{PE})$ to furnish $\mathbf{1 0}$ as a brownish solid, yield $83 \%, 4.11 \mathrm{~g}, \mathrm{mp} 164-166^{\circ} \mathrm{C}$; IR $\left(v_{\max }, \mathrm{cm}^{-1}\right): 3320,1727,1542,1369$, 1282, 1171, 1156, 1088, 1077, 810, 725. ${ }^{1} \mathrm{H} \mathrm{NMR}\left(\mathrm{CDCl}_{3}\right): \delta_{\mathrm{H}} 7.50\left(2 \mathrm{H}, \mathrm{td}, J_{1} 7.5 \mathrm{~Hz}, J_{2} 1.5\right.$ $\mathrm{Hz}), 7.62\left(1 \mathrm{H}, \mathrm{tt}, J_{1} 7.5 \mathrm{~Hz}, J_{2} 1.5 \mathrm{~Hz}\right), 8.00\left(2 \mathrm{H}, \mathrm{td}, J_{1} 8 \mathrm{~Hz}, J_{2} 1.5 \mathrm{~Hz}\right), 8.06(1 \mathrm{H}, \mathrm{s}), 8.21(1 \mathrm{H}$, $\mathrm{d}, J 9 \mathrm{~Hz}), 8.24(1 \mathrm{H}, \mathrm{d}, J 9 \mathrm{~Hz}), 8.30\left(1 \mathrm{H}\right.$, br s). ${ }^{13} \mathrm{C} \mathrm{NMR}\left(\mathrm{CDCl}_{3}\right): \delta_{\mathrm{C}} 113.9,124.3,127.7$, 129.4, 134.7, 143.7 (all $\mathrm{CH}_{\text {arom}}$ ), 82.8, 130.5, 132.8, 137.0, 137.8 (all $\mathrm{C}_{\text {arom }}$ ), $155.1\left(\mathrm{NHCOCF}_{3}\right)$. MS (ESI) calcd for $\mathrm{C}_{15} \mathrm{H}_{9} \mathrm{IN}_{3} \mathrm{O}_{3} \mathrm{~F}_{3} \mathrm{~S}\left(\mathrm{M}^{+}\right)$494.9361; found 494.9340. MS (EI), $m / z(\%)=495$ (40) $\left(\mathrm{M}^{+}\right), 368$ (100) (M-I). Anal. Calcd for $\mathrm{C}_{15} \mathrm{H}_{9} \mathrm{IN}_{3} \mathrm{O}_{3} \mathrm{~F}_{3} \mathrm{~S}: \mathrm{C}, 36.36 ; \mathrm{H}, 1.82 ; \mathrm{N}, 8.48 \%$. Found: C, 36.41; H, 1.80; N, 8.51\%.

General Procedure for the synthesis of 7-H/substituted 3-phenylsulfonyl-3,6-dihydropyrrolo[3,2-e]indazoles (12a-h). A mixture of $10(0.25 \mathrm{~g}, 0.5 \mathrm{mmol})$, the alkyne 11a-h $(0.7$ $\mathrm{mmol}), \mathrm{Pd}\left(\mathrm{Ph}_{3} \mathrm{P}\right)_{2} \mathrm{Cl}_{2}(35 \mathrm{mg} ; 10 \mathrm{~mol} \%), \mathrm{CuI}(19 \mathrm{mg} ; 10 \mathrm{~mol} \%)$ and $\mathrm{Et}_{3} \mathrm{~N}(0.7 \mathrm{~mL} ; 5 \mathrm{mmol})$ in DMF $(3 \mathrm{~mL})$ was stirred at $100{ }^{\circ} \mathrm{C}$ in argon atmosphere until $(5-10 \mathrm{~h})$ the reaction was complete (the reaction darkened in color). It was cooled to rt, filtered through a bed of Celite ${ }^{\circledR}$ and washed with EtOAc $(3 \times 15 \mathrm{~mL})$. The combined organic layer was washed with brine and then $\mathrm{H}_{2} \mathrm{O}$, 
dried $\left(\mathrm{Na}_{2} \mathrm{SO}_{4}\right)$ and the solvent distilled off. The residue obtained was purified by $\mathrm{CC}$ and crystallized from EtOAc/PE mixture.

3-Phenylsulfonyl-3,6-dihydropyrrolo[3,2-e]indazole (12a). Pale yellow solid, yield 83\%, $0.123 \mathrm{~g}, \mathrm{mp} 204-206^{\circ} \mathrm{C}(\mathrm{dec})$; IR $\left(v_{\max }, \mathrm{cm}^{-1}\right)$ : 3307, 1584, 1480, 1447, 1375, 1185, 1117, 887, 771, 727, 682. ${ }^{1} \mathrm{H}$ NMR (DMSO-d $): \delta_{\mathrm{H}} 6.75(1 \mathrm{H}, \mathrm{d}, J 2 \mathrm{~Hz}), 7.50\left(2 \mathrm{H}, \mathrm{dd}, J_{1} 7.5 \mathrm{~Hz}, J_{2} 2 \mathrm{~Hz}\right)$, $7.53(1 \mathrm{H}, \mathrm{d}, J 1 \mathrm{~Hz}), 7.62(1 \mathrm{H}, \mathrm{t}, J 7.5 \mathrm{~Hz}), 7.71(1 \mathrm{H}, \mathrm{d}, J 9 \mathrm{~Hz}), 7.85\left(2 \mathrm{H}, \mathrm{dd}, J_{1} 7.5 \mathrm{~Hz}, J_{2} 1.5\right.$ $\mathrm{Hz}), 7.87(1 \mathrm{H}, \mathrm{d}, J 9 \mathrm{~Hz}), 8.69(1 \mathrm{H}, \mathrm{s}), 11.65(1 \mathrm{H}, \mathrm{s}) .{ }^{13} \mathrm{C}$ NMR (DMSO- $\left.d_{6}\right): \delta_{\mathrm{C}} 100.7,106.1$, 115.6, 127.0, 127.2, 130.1, 135.0, 141.9 (all $\mathrm{CH}_{\text {arom}}$ ), 118.8, 119.0, 132.9, 136.6, 137.0 (all $\left.\mathrm{C}_{\text {arom}}\right)$. MS (ESI) calcd for $\mathrm{C}_{15} \mathrm{H}_{11} \mathrm{~N}_{3} \mathrm{O}_{2} \mathrm{SNa}(\mathrm{M}+\mathrm{Na}) 320.0470$; found: 320.0471 .

7-n-Butyl-3-phenylsulfonyl-3,6-dihydropyrrolo[3,2-e]indazole (12b). Colorless solid, yield $88 \%, 0.155 \mathrm{~g}, \mathrm{mp} 214-216{ }^{\circ} \mathrm{C}(\mathrm{dec})$; IR $\left(v_{\max }, \mathrm{cm}^{-1}\right): 3359,1589,1370,1186,726 .{ }^{1} \mathrm{H} \mathrm{NMR}$ (DMSO- $\left.d_{6}\right): \delta_{\mathrm{H}} 0.88(3 \mathrm{H}, \mathrm{t}, J 7.5 \mathrm{~Hz}), 1.31(2 \mathrm{H}$, sextet, $J 7.5 \mathrm{~Hz}), 1.65(2 \mathrm{H}$, quintet, $J 7.5 \mathrm{~Hz})$, $2.74(2 \mathrm{H}, \mathrm{t}, J 7.5 \mathrm{~Hz}), 6.47(1 \mathrm{H}, \mathrm{s}), 7.52(2 \mathrm{H}, \mathrm{t}, J 7.5 \mathrm{~Hz}), 7.59(1 \mathrm{H}, \mathrm{d}, J 9 \mathrm{~Hz}), 7.63(1 \mathrm{H}, \mathrm{t}, J 7.5$ $\mathrm{Hz}), 7.78(1 \mathrm{H}, \mathrm{d}, J 9 \mathrm{~Hz}), 7.84(2 \mathrm{H}, \mathrm{d}, J 8 \mathrm{~Hz}), 8.64(1 \mathrm{H}, \mathrm{s}), 11.48(1 \mathrm{H}, \mathrm{s}) .{ }^{13} \mathrm{C}$ NMR (DMSO$\left.d_{6}\right): \delta_{\mathrm{C}} 14.1\left(\mathrm{CH}_{3}\right), 22.2,27.7,31.4\left(\right.$ all $\left.\mathrm{CH}_{2}\right), 98.1,104.8,114.8,127.2$, 130.0, 135.0, 142.0 (all $\mathrm{CH}_{\text {arom}}$ ), 118.3, 119.7, 132.7, 136.5, 137.0, 142.4 (all $\mathrm{C}_{\text {arom}}$ ). MS (EI) $m / z(\%) 353(100)\left(\mathrm{M}^{+}\right)$, $311(80)\left(\mathrm{M}-\mathrm{C}_{3} \mathrm{H}_{6}\right), 310(72)\left(\mathrm{M}-\mathrm{C}_{3} \mathrm{H}_{7}\right), 212$ (40) $\left(\mathrm{M}-\mathrm{SO}_{2} \mathrm{Ph}\right)$. Anal. Calcd for $\mathrm{C}_{19} \mathrm{H}_{19} \mathrm{~N}_{3} \mathrm{O}_{2} \mathrm{~S}$ : C, 64.57; H, 5.42; N, 11.89\%. Found: C, 64.52; H, 5.44; N, 11.88\%.

7-n-Pentyl-3-phenylsulfonyl-3,6-dihydropyrrolo[3,2-e]indazole (12c). Colorless solid, yield $82 \%, 0.151 \mathrm{~g}, \mathrm{mp} 186-188{ }^{\circ} \mathrm{C}(\mathrm{dec})$; IR $\left(v_{\max }, \mathrm{cm}^{-1}\right): 3338,1584,1523,1451,1369,1184,1118$, 808, 727. ${ }^{1} \mathrm{H} \mathrm{NMR}\left(\mathrm{CDCl}_{3}\right): \delta_{\mathrm{H}} 0.90(3 \mathrm{H}, \mathrm{t}, J 7.0 \mathrm{~Hz}), 1.32-1.40(4 \mathrm{H}, \mathrm{m}), 1.70(2 \mathrm{H}$, quintet, $J 7.5$ Hz), $2.83(2 \mathrm{H}, \mathrm{t}, J 7.5 \mathrm{~Hz}), 6.47(1 \mathrm{H}, \mathrm{s}), 7.41(2 \mathrm{H}, \mathrm{t}, J 8 \mathrm{~Hz}), 7.50(1 \mathrm{H}, \mathrm{d}, J 9 \mathrm{~Hz}), 7.52(1 \mathrm{H}, \mathrm{t}, J$ $7.5 \mathrm{~Hz}), 7.96(2 \mathrm{H}, \mathrm{d}, J 8.5 \mathrm{~Hz}), 8.03(1 \mathrm{H}, \mathrm{d}, J 9 \mathrm{~Hz}), 8.72(1 \mathrm{H}$, br s$), 8.98(1 \mathrm{H}, \mathrm{s}) .{ }^{13} \mathrm{C}$ NMR $\left(\mathrm{CDCl}_{3}\right): \delta_{\mathrm{C}} 13.9\left(\mathrm{CH}_{3}\right), 22.4,28.5,28.9,31.3(\mathrm{all} \mathrm{CH}), 98.5,107.0,113.6,127.3,129.1,133.9$, 138.3 (all $\mathrm{CH}_{\text {arom}}$ ), 105.9, 113.5, 129.0, 133.8, 140.5, 141.6 (all $\mathrm{C}_{\text {arom}}$ ). MS (ESI) calcd for $\mathrm{C}_{20} \mathrm{H}_{21} \mathrm{~N}_{3} \mathrm{O}_{2} \mathrm{SNa}(\mathrm{M}+\mathrm{Na})$ 390.1252; found 390.1198. Anal. Calcd for $\mathrm{C}_{20} \mathrm{H}_{21} \mathrm{~N}_{3} \mathrm{O}_{2} \mathrm{~S}$ : C, 65.40; $\mathrm{H}$, 5.72; N, 11.44\%. Found: C, 65.45; H, 5.74; N, 11.46\%.

7-n-Hexyl-3-phenylsulfonyl-3,6-dihydropyrrolo[3,2-e]indazole (12d). Colorless solid, yield $85 \%, 0.162 \mathrm{~g}, \mathrm{mp} 180-182{ }^{\circ} \mathrm{C}(\mathrm{dec})$; IR $\left(v_{\max }, \mathrm{cm}^{-1}\right): 3319,1583,1375,1189,1074,726 .{ }^{1} \mathrm{H}$ NMR (DMSO- $\left.d_{6}\right): \delta_{\mathrm{H}} 0.80(3 \mathrm{H}, \mathrm{t}, J 7.0 \mathrm{~Hz}), 1.24(6 \mathrm{H}, \mathrm{m}), 1.63(2 \mathrm{H}$, quintet, $J 7 \mathrm{~Hz}), 2.74(2 \mathrm{H}$, t, $J 7.5 \mathrm{~Hz}), 6.46(1 \mathrm{H}, \mathrm{s}),, 7.53(2 \mathrm{H}, \mathrm{t}, J 7.5 \mathrm{~Hz}), 7.64(1 \mathrm{H}, \mathrm{t}, J 7 \mathrm{~Hz}), 7.65(1 \mathrm{H}, \mathrm{d}, J 7.5 \mathrm{~Hz}), 7.87$ $(2 \mathrm{H}, \mathrm{d}, J 7.5 \mathrm{~Hz}), 7.88(1 \mathrm{H}, \mathrm{d}, J 7.5 \mathrm{~Hz}), 8.93(1 \mathrm{H}, \mathrm{s}), 12.04(1 \mathrm{H}, \mathrm{s},) .{ }^{13} \mathrm{C}$ NMR $\left(\mathrm{DMSO}-d_{6}\right): \delta_{\mathrm{C}}$ $14.3\left(\mathrm{CH}_{3}\right), 22.4,28.1,28.5,29.1,31.3\left(\right.$ all $\left.\mathrm{CH}_{2}\right), 98.0,106.1,115.3,127.2,130.1,135.2,138.5$ (all $\mathrm{CH}_{\text {arom}}$ ), 118.1, 121.7, 133.1, 136.9, 137.1, 142.9 (all $\mathrm{C}_{\text {arom}}$ ). MS (ESI) calcd for $\mathrm{C}_{21} \mathrm{H}_{24} \mathrm{~N}_{3} \mathrm{O}_{2} \mathrm{~S}(\mathrm{M}+\mathrm{H})$ 382.1589; found: 382.1592. Anal. Calcd for $\mathrm{C}_{21} \mathrm{H}_{23} \mathrm{~N}_{3} \mathrm{O}_{2} \mathrm{~S}$ : C, 66.14; $\mathrm{H}$, 6.04; N, 11.02\%. Found: C, 66.18; H, 6.07; N, 11.07\%.

7-(3-Cyanopropyl)-3-phenylsulfonyl-3,6-dihydropyrrolo[3,2-e]indazole (12e). Colorless solid, yield 94\%, $0.171 \mathrm{~g}, \mathrm{mp} 258-260{ }^{\circ} \mathrm{C}(\mathrm{dec})$; IR $\left(v_{\max }, \mathrm{cm}^{-1}\right): 3345,2241,1588,1538,1445$, $1366,1188,1105,728,689 .{ }^{1} \mathrm{H}$ NMR (DMSO- $\left.d_{6}\right): \delta_{\mathrm{H}} 1.96(2 \mathrm{H}$, quintet, $J 7 \mathrm{~Hz}), 2.51(2 \mathrm{H}, \mathrm{t}, J$ $7.5 \mathrm{~Hz}), 2.85(2 \mathrm{H}, \mathrm{t}, J 7.5 \mathrm{~Hz}), 6.52(1 \mathrm{H}, \mathrm{s}), 7.51(2 \mathrm{H}, \mathrm{t}, J 8 \mathrm{~Hz}), 7.60(1 \mathrm{H}, \mathrm{d}, J 8.5 \mathrm{~Hz}), 7.62$ 
$(1 \mathrm{H}, \mathrm{t}, J 8 \mathrm{~Hz}), 7.79(1 \mathrm{H}, \mathrm{d}, J 9 \mathrm{~Hz}), 7.83(2 \mathrm{H}, \mathrm{t}, J 8 \mathrm{~Hz}), 8.64(1 \mathrm{H}, \mathrm{s}), 11.58(1 \mathrm{H}, \mathrm{s}) .{ }^{13} \mathrm{C}$ NMR $\left(\mathrm{DMSO}-d_{6}\right): \delta_{\mathrm{C}} 16.2,25.0,27.0\left(\right.$ all $\left.\mathrm{CH}_{2}\right), 98.7,105.2,114.9,127.2,130.0,132.9,135.0$ (all $\left.\mathrm{CH}_{\text {arom }}\right), 118.4,119.6,120.8,136.5,137.0,140.3,142.0\left(\mathrm{CN}+\right.$ rest $\left.\mathrm{C}_{\text {arom}}\right) . \mathrm{MS}$ (ESI) calcd for $\mathrm{C}_{19} \mathrm{H}_{16} \mathrm{~N}_{4} \mathrm{O}_{2} \mathrm{SNa}(\mathrm{M}+\mathrm{Na}) 387.0892$; found: 387.0854. Anal. Calcd for $\mathrm{C}_{19} \mathrm{H}_{16} \mathrm{~N}_{4} \mathrm{O}_{2} \mathrm{~S}: \mathrm{C}, 62.64 ; \mathrm{H}$, 4.39; N, 15.38\%. Found: C, 62.69; H, 4.37; N, 15.40\%.

7-Phenyl-3-phenylsulfonyl-3,6-dihydropyrrolo[3,2-e]indazole (12f). Colorless solid, yield 93\%, 0.174 g, mp 238-240 ${ }^{\circ} \mathrm{C}(\mathrm{dec})$; IR ( $\left.v_{\max }, \mathrm{cm}^{-1}\right): 3349,1589,1476,1446,1187,1114,766$, 751, 726, 685. ${ }^{1} \mathrm{H}$ NMR (DMSO- $\left.d_{6}\right): \delta_{\mathrm{H}} 7.21(1 \mathrm{H}, \mathrm{s}), 7.31(1 \mathrm{H}, \mathrm{t}, J 7.5 \mathrm{~Hz}), 7.46(2 \mathrm{H}, \mathrm{t}, J 7.5$ $\mathrm{Hz}), 7.53(2 \mathrm{H}, \mathrm{t}, J 7.5 \mathrm{~Hz}), 7.64(1 \mathrm{H}, \mathrm{t}, J 7 \mathrm{~Hz}), 7.71(1 \mathrm{H}, \mathrm{d}, J 9 \mathrm{~Hz}), 7.85(2 \mathrm{H}, \mathrm{d}, J 8.5 \mathrm{~Hz}), 7.87$ $(2 \mathrm{H}, \mathrm{d}, J 9 \mathrm{~Hz}), 7.89(1 \mathrm{H}, \mathrm{d}, J 9.5 \mathrm{~Hz}), 8.70(1 \mathrm{H}, \mathrm{s}), 12.10(1 \mathrm{H}, \mathrm{s}) .{ }^{13} \mathrm{C}$ NMR $\left(\mathrm{DMSO}-d_{6}\right): \delta_{\mathrm{C}}$ 98.3, 106.7, 115.3, 125.6, 127.0, 128.3, 129.5, 130.1, 135.1, 141.8 (all $\mathrm{CH}_{\text {arom }}$ ), 118.6, 120.1, 132.1, 134.1, 136.9, 137.0, 139.4 (all $\mathrm{C}_{\text {arom}}$ ). MS (ESI) calcd for $\mathrm{C}_{21} \mathrm{H}_{15} \mathrm{~N}_{3} \mathrm{O}_{2} \mathrm{SNa}(\mathrm{M}+\mathrm{Na})$ 396.0783; found: 396.0773. Anal. Calcd for $\mathrm{C}_{21} \mathrm{H}_{15} \mathrm{~N}_{3} \mathrm{O}_{2} \mathrm{~S}$ : C, 67.56; H, 4.02; N, 11.26\%. Found: C, 67.52; H, 4.03; N, $11.29 \%$.

7-(4-Methylphenyl)-3-phenylsulfonyl-3,6-dihydropyrrolo[3,2-e]indazole $\quad(12 \mathrm{~g})$. Colorless solid, yield $88 \%, 0.170 \mathrm{~g}, \mathrm{mp} 222-224{ }^{\circ} \mathrm{C}(\mathrm{dec})$; IR $\left(v_{\max }, \mathrm{cm}^{-1}\right): 3379,1589,1498,1474,1449$, 1367, 1182, 1109, 807, 783, 727. ${ }^{1} \mathrm{H}$ NMR (DMSO-d $): \delta_{\mathrm{H}} 2.47(3 \mathrm{H}, \mathrm{s}), 7.32(1 \mathrm{H}, \mathrm{s}), 7.43(2 \mathrm{H}$, d, J $8.0 \mathrm{~Hz}), 7.70(2 \mathrm{H}, \mathrm{t}, J 7.5 \mathrm{~Hz}), 7.80(1 \mathrm{H}, \mathrm{t}, J 7.5 \mathrm{~Hz}), 7.88(1 \mathrm{H}, \mathrm{d}, J 9 \mathrm{~Hz}), 7.92(2 \mathrm{H}, \mathrm{d}, J 8$ $\mathrm{Hz}), 8.05(2 \mathrm{H}, \mathrm{d}, J 7.5 \mathrm{~Hz}), 8.06(1 \mathrm{H}, \mathrm{d}, J 9.5 \mathrm{~Hz}), 8.87(1 \mathrm{H}, \mathrm{s}), 12.22(1 \mathrm{H}, \mathrm{s}) .{ }^{13} \mathrm{C}$ NMR (DMSO- $\left.d_{6}\right): \delta_{\mathrm{C}} 21.2\left(\mathrm{CH}_{3}\right), 97.7,106.3,115.2,125.5,127.2,130.0,130.1,135.0,141.9$ (all $\mathrm{CH}_{\text {arom}}$ ), 118.8, 120.2, 129.4, 133.9, 136.9, 137.0, 137.7, 139.6 (all $\mathrm{C}_{\text {arom }}$ ). MS (ESI) calcd for $\mathrm{C}_{22} \mathrm{H}_{17} \mathrm{~N}_{3} \mathrm{O}_{2} \mathrm{SNa}(\mathrm{M}+\mathrm{Na}) 410.0939$; found: 410.0932. Anal. Calcd for $\mathrm{C}_{22} \mathrm{H}_{17} \mathrm{~N}_{3} \mathrm{O}_{2} \mathrm{~S}: \mathrm{C}, 68.22 ; \mathrm{H}$, 4.39; N, 10.85\%. Found: C, 68.16; H, 4.40; N, 10.89\%.

7-(4-Methoxyphenyl)-3-phenylsulfonyl-3,6-dihydropyrrolo[3,2-e]indazole (12h). Colorless solid, yield 87\%, $0.175 \mathrm{~g}, \mathrm{mp} 218-220^{\circ} \mathrm{C}$; IR $\left(v_{\max }, \mathrm{cm}^{-1}\right): 3448,1608,1545,1497,1379,1254$, $1183,1111,1077,1023,832,790,730,683 .{ }^{1} \mathrm{H}$ NMR (DMSO-d $): \delta_{\mathrm{H}} 3.94(3 \mathrm{H}, \mathrm{s}), 7.21(2 \mathrm{H}$, dd, $\left.J_{1} 8.0 \mathrm{~Hz}, J_{2} 1 \mathrm{~Hz}\right), 7.25(1 \mathrm{H}, \mathrm{s}), 7.70(2 \mathrm{H}, \mathrm{t}, J 8 \mathrm{~Hz}), 7.80\left(1 \mathrm{H}, \mathrm{dt}, J_{1} 7.5 \mathrm{~Hz}, J_{2} 1 \mathrm{~Hz}\right), 7.87(1 \mathrm{H}$, $\mathrm{d}, J 8.5 \mathrm{~Hz}), 7.96(2 \mathrm{H}, \mathrm{d}, J 8 \mathrm{~Hz}), 8.02-8.08(3 \mathrm{H}, \mathrm{m}), 8.87(1 \mathrm{H}, \mathrm{s}), 12.17(1 \mathrm{H}, \mathrm{s}) .{ }^{13} \mathrm{C}$ NMR $\left(\mathrm{DMSO}-d_{6}\right): \delta_{\mathrm{C}} 55.6\left(\mathrm{OCH}_{3}\right), 97.1,106.0,114.9,115.1,127.0,127.2,130.1,135.0,141.9$ (all $\mathrm{CH}_{\text {arom}}$ ), 118.5, 120.3, 124.8, 133.9, 136.9, 137.1, 136.9, 159.5 (all $\mathrm{C}_{\text {arom }}$ ). MS (ESI) calcd for $\mathrm{C}_{22} \mathrm{H}_{17} \mathrm{~N}_{3} \mathrm{O}_{3} \mathrm{SNa}(\mathrm{M}+\mathrm{Na}) 426.0889$; found: 426.0889. Anal. Calcd for $\mathrm{C}_{22} \mathrm{H}_{17} \mathrm{~N}_{3} \mathrm{O}_{3} \mathrm{~S}: \mathrm{C}, 65.51 ; \mathrm{H}$, 4.22; N, 10.42\%. Found: C, 65.57; H, 4.23; N, 10.44\%.

General procedure for $\mathbf{N ( 3 )}$-deprotection of $12 \mathbf{a}-\mathbf{h}$. To a solution of $\mathbf{1 2 a}-\mathbf{h}(1 \mathrm{mmol})$ in $\mathrm{MeOH}$ $(30 \mathrm{~mL})$ was added an aqueous solution $(10 \mathrm{~mL})$ of $\mathrm{K}_{2} \mathrm{CO}_{3}(0.55 \mathrm{~g} ; 4 \mathrm{mmol})$, and the solution was refluxed for $2 \mathrm{~h}$. It was diluted with $\mathrm{H}_{2} \mathrm{O}(40 \mathrm{~mL}), \mathrm{MeOH}$ distilled off and the solution extracted with EtOAc $(3 \times 30 \mathrm{~mL})$. The combined organic layer was washed with brine and then $\mathrm{H}_{2} \mathrm{O}$, dried $\left(\mathrm{Na}_{2} \mathrm{SO}_{4}\right)$ and the solvent distilled off. The residue was purified by $\mathrm{CC}$ to furnish 13ah. All the products were crystallised from EtOAc/PE.

3,6-Dihydropyrrolo[3,2-e]indazole (13a). Colorless solid, yield 87\%, 0.137 g, mp 162-164 ${ }^{\circ} \mathrm{C}$ (dec); IR ( $\left.v_{\max }, \mathrm{cm}^{-1}\right): 3433,3327,1505,937,771 .{ }^{1} \mathrm{H} \mathrm{NMR}\left(400 \mathrm{MHz}, \mathrm{CDCl}_{3}\right): \delta_{\mathrm{H}} 6.83(1 \mathrm{H}, \mathrm{d}$, 
$J 2 \mathrm{~Hz}), 7.28(1 \mathrm{H}, \mathrm{d}, J 2 \mathrm{~Hz}), 7.29(1 \mathrm{H}, \mathrm{d}, J 8.8 \mathrm{~Hz}), 7.45(1 \mathrm{H}, \mathrm{d}, J 8.8 \mathrm{~Hz}), 8.23(1 \mathrm{H}, \mathrm{s}), 8.94$ (1H, br s). ${ }^{13} \mathrm{C} \mathrm{NMR}\left(100 \mathrm{MHz}, \mathrm{CDCl}_{3}\right): \delta_{\mathrm{C}} 100.5,104.6,112.7,123.2,131.8$ (all $\left.\mathrm{CH}_{\text {arom }}\right), 115.8$, 118.0, 130.3, 136.9 (all $\mathrm{C}_{\text {arom }}$ ). MS (ESI) calcd for $\mathrm{C}_{9} \mathrm{H}_{8} \mathrm{~N}_{3}(\mathrm{M}+\mathrm{H})$ 158.0712; found: 158.0710. Anal. Calcd for $\mathrm{C}_{9} \mathrm{H}_{7} \mathrm{~N}_{3}$ : C, 68.78; H, 4.49; N, 26.74\%. Found: C, 68.80; H, 4.48; N, 26.77\%.

7-n-Butyl-3,6-dihydropyrrolo[3,2-e]indazole (13b). Colorless solid, yield 86\%, $0.183 \mathrm{~g}, \mathrm{mp}$ $134-136{ }^{\circ} \mathrm{C}(\mathrm{dec})$; IR $\left(v_{\max }, \mathrm{cm}^{-1}\right): 3371,3202,3178,1546,1344,938,780 .{ }^{1} \mathrm{H} \mathrm{NMR}\left(\mathrm{CDCl}_{3}\right): \delta_{\mathrm{H}}$ $0.96(3 \mathrm{H}, \mathrm{t}, J 7.5 \mathrm{~Hz}), 1.43(2 \mathrm{H}$, sextet, $J 7.5 \mathrm{~Hz}), 1.73(2 \mathrm{H}$, quintet, $J 7.5 \mathrm{~Hz}), 2.81(2 \mathrm{H}, \mathrm{t}, J 7.5$ $\mathrm{Hz}), 6.54(1 \mathrm{H}, \mathrm{s}), 7.18(1 \mathrm{H}, \mathrm{d}, J 9 \mathrm{~Hz}), 7.34(1 \mathrm{H}, \mathrm{d}, J 8.5 \mathrm{~Hz}), 8.24(1 \mathrm{H}, \mathrm{s}), 8.34(1 \mathrm{H}, \mathrm{s}), 10.47$ $\left(1 \mathrm{H}\right.$, br s). ${ }^{13} \mathrm{C} \mathrm{NMR}\left(\mathrm{CDCl}_{3}\right): \delta_{\mathrm{C}} 13.9\left(\mathrm{CH}_{3}\right), 22.4,28.0,31.5\left(\right.$ all $\left.\mathrm{CH}_{2}\right), 98.7,103.2,112.3$, 132.8 (all $\mathrm{CH}_{\text {arom}}$ ), 115.8, 119.3, 129.8, 136.9, 139.4 (all $\mathrm{C}_{\text {arom}}$ ). MS (ESI) calcd for $\mathrm{C}_{13} \mathrm{H}_{16} \mathrm{~N}_{3}$ $(\mathrm{M}+\mathrm{H})$ 214.1343; found: 214.1349. Anal. Calcd for $\mathrm{C}_{13} \mathrm{H}_{15} \mathrm{~N}_{3}$ : C, 73.21; H, 7.09; N, 19.70\%. Found: C, 73.25; H, 7.10; N, 19.73\%.

7-n-Pentyl-3,6-dihydropyrrolo[3,2-e]indazole (13c). Colorless solid, yield 85\%, $0.195 \mathrm{~g}$, mp $168-170{ }^{\circ} \mathrm{C}$; IR $\left(v_{\max }, \mathrm{cm}^{-1}\right): 3271,3205,1530,1464,1378,945,783 .{ }^{1} \mathrm{H}$ NMR (DMSO- $\left.d_{6}\right): \delta_{\mathrm{H}}$ $0.83(3 \mathrm{H}, \mathrm{t}, J 7 \mathrm{~Hz}), 1.27-1.29(4 \mathrm{H}, \mathrm{m}), 1.64(2 \mathrm{H}$, quintet, $J 7 \mathrm{~Hz}), 2.72(2 \mathrm{H}, \mathrm{t}, J 7.5 \mathrm{~Hz}), 6.37$ $(1 \mathrm{H}, \mathrm{s}), 7.23(1 \mathrm{H}, \mathrm{d}, J 9 \mathrm{~Hz}), 7.35(1 \mathrm{H}, \mathrm{d}, J 9 \mathrm{~Hz}), 8.46(1 \mathrm{H}, \mathrm{s}), 11.64(1 \mathrm{H}, \mathrm{s}), 13.04(1 \mathrm{H}, \mathrm{s}) .{ }^{13} \mathrm{C}$ NMR (DMSO- $\left.d_{6}\right): \delta_{\mathrm{C}} 14.3\left(\mathrm{CH}_{3}\right), 22.2,28.1,29.0,31.1\left(\right.$ all $\left.\mathrm{CH}_{2}\right), 98.1,104.7,112.8,129.3$ (all $\mathrm{CH}_{\text {arom}}$ ), 115.3, 121.1, 130.4, 137.3, 139.6 (all $\mathrm{C}_{\text {arom}}$ ). MS (ESI) calcd for $\mathrm{C}_{14} \mathrm{H}_{18} \mathrm{~N}_{3}(\mathrm{M}+\mathrm{H})$ 228.1500; found: 228.1500. Anal. Calcd for $\mathrm{C}_{14} \mathrm{H}_{17} \mathrm{~N}_{3}$ : C, 74.00; H, 7.49; N, 18.5\%. Found: C, $74.15 ; \mathrm{H}, 7.47 ; \mathrm{N}, 18.55 \%$.

7-n-Hexyl-3,6-dihydropyrrolo[3,2-e]indazole (13d). Colorless solid, yield 88\%, $0.212 \mathrm{~g}, \mathrm{mp}$ 134-136 ${ }^{\circ} \mathrm{C}$; IR $\left(v_{\max }, \mathrm{cm}^{-1}\right): 3379,3214,1531,1449,1342,1167,1115,1038,943,843,776$, 684. ${ }^{1} \mathrm{H}$ NMR $\left(\mathrm{CDCl}_{3}\right): \delta_{\mathrm{H}} 0.89(3 \mathrm{H}, \mathrm{t}, J 6.5 \mathrm{~Hz}), 1.27-1.36(4 \mathrm{H}, \mathrm{m}), 1.39$ (2H, quintet, $\left.J 7 \mathrm{~Hz}\right)$, $1.70(2 \mathrm{H}$, quintet, $J 7.5 \mathrm{~Hz}), 2.83(2 \mathrm{H}, \mathrm{t}, J 7.5 \mathrm{~Hz}), 6.54(1 \mathrm{H}, \mathrm{s}), 7.24(1 \mathrm{H}, \mathrm{d}, J 8.5 \mathrm{~Hz}), 7.34(1 \mathrm{H}$, d, $J 8.5 \mathrm{~Hz}), 8.73(1 \mathrm{H}, \mathrm{s}), 8.83(1 \mathrm{H}, \mathrm{s}) .{ }^{13} \mathrm{C} \mathrm{NMR}\left(\mathrm{CDCl}_{3}\right): \delta_{\mathrm{C}} 14.0\left(\mathrm{CH}_{3}\right), 22.4,28.3,28.7,28.7$, $31.4\left(\right.$ all $\mathrm{CH}_{2}$ ), 97.8, 104.4, 112.3, 130.1 (all $\mathrm{CH}_{\text {arom}}$ ), 115.3, 121.2, 129.8, 137.4, 139.5 (all $\mathrm{C}_{\text {arom}}$ ). MS (ESI) calcd for $\mathrm{C}_{15} \mathrm{H}_{20} \mathrm{~N}_{3}(\mathrm{M}+\mathrm{H})$ 242.1657; found: 242.1667. Anal. Calcd for $\mathrm{C}_{15} \mathrm{H}_{19} \mathrm{~N}_{3}$ : C, 74.69; H, 7.88; N, 17.43\%. Found: C, 74.60; H, 7.85; N, 17.46\%.

7-(3-Cyanopropyl)-3,6-dihydropyrrolo[3,2-e]indazole (13e). Colorless solid, yield 90\%, 0.202 g, mp 130-132 ${ }^{\circ} \mathrm{C}$; IR $\left(v_{\max }, \mathrm{cm}^{-1}\right): 3381,3330,2251,1626,1600,1551,1437,1327,945,783$. ${ }^{1} \mathrm{H}$ NMR (DMSO- $\left.d_{6}\right): \delta_{\mathrm{H}} 2.16(2 \mathrm{H}, \mathrm{t}, J 7 \mathrm{~Hz}), 2.68(2 \mathrm{H}, \mathrm{t}, J 6.5 \mathrm{~Hz}), 3.02(2 \mathrm{H}, \mathrm{t}, J 6.5 \mathrm{~Hz}), 6.61$ $(1 \mathrm{H}, \mathrm{s}), 7.32(1 \mathrm{H}, \mathrm{d}, J 8.5 \mathrm{~Hz}), 7.49(1 \mathrm{H}, \mathrm{d}, J 8.5 \mathrm{~Hz}), 8.24(1 \mathrm{H}, \mathrm{s}), 11.33(1 \mathrm{H}, \mathrm{s}), 13.00(1 \mathrm{H}, \mathrm{s})$. ${ }^{13} \mathrm{C}$ NMR (DMSO- $\left.d_{6}\right): \delta_{\mathrm{C}} 16.3,25.2,27.1\left(\right.$ all $\left.\mathrm{CH}_{2}\right), 98.7,103.6,112.5,132.0$ (all $\mathrm{CH}_{\text {arom}}$ ), $118.7,115.7,120.9,130.3,132.0,137.5\left(\mathrm{CN}+\right.$ rest $\left.\mathrm{C}_{\text {arom }}\right) . \mathrm{MS}(\mathrm{ESI})$ calcd for $\mathrm{C}_{13} \mathrm{H}_{13} \mathrm{~N}_{4}(\mathrm{M}+\mathrm{H})$ 225.1146; found: 225.1105. Anal. Calcd for $\mathrm{C}_{13} \mathrm{H}_{12} \mathrm{~N}_{4}$ : C, 69.64; H, 5.36; N, 25.00\%. Found: C, $69.60 ; \mathrm{H}, 5.37$; N, 24.95\%.

7-Phenyl-3,6-dihydropyrrolo[3,2-e]indazole (13f). Pale yellow solid, yield 87\%, $0.203 \mathrm{~g}, \mathrm{mp}$ 246-248 ${ }^{\circ} \mathrm{C}$; IR $\left(v_{\max }, \mathrm{cm}^{-1}\right): 3434,3128,1624,1596,1475,1439,1379,1164,952,788,754$, 689. ${ }^{1} \mathrm{H}$ NMR (DMSO- $\left.d_{6}\right): \delta_{\mathrm{H}} 7.33(1 \mathrm{H}, \mathrm{s}), 7.44(1 \mathrm{H}, \mathrm{d}, J 8 \mathrm{~Hz}), 7.60(2 \mathrm{H}, \mathrm{t}, J 8 \mathrm{~Hz}), 7.61(2 \mathrm{H}, \mathrm{t}$, $J 7.5 \mathrm{~Hz}), 8.03(2 \mathrm{H}, \mathrm{d}, J 7.5 \mathrm{~Hz}), 8.33(1 \mathrm{H}, \mathrm{s}), 11.91(1 \mathrm{H}, \mathrm{s}), 13.13(1 \mathrm{H}, \mathrm{s}) .{ }^{13} \mathrm{C}$ NMR (DMSO- 
$\left.d_{6}\right): \delta_{\mathrm{C}} 98.5,105.3,112.8,125.0,127.4,129.4,131.8$ (all $\mathrm{CH}_{\text {arom}}$ ), 115.8, 119.4, 131.9, 132.9, 136.6, 137.0 (all $\mathrm{C}_{\text {arom }}$ ). MS (ESI) calcd for $\mathrm{C}_{15} \mathrm{H}_{11} \mathrm{~N}_{3}\left(\mathrm{M}^{+}\right)$233.0953; found: 233.0900. Anal. Calcd for $\mathrm{C}_{15} \mathrm{H}_{11} \mathrm{~N}_{3}$ : C, 77.25; H, 4.72; N, 18.03\%. Found: C, 77.25; 4.70; N, 18.06\%.

7-(4-Methylphenyl)-3,6-dihydropyrrolo[3,2-e]indazole (13g). Colorless solid, yield 91\%, $0.225 \mathrm{~g}, \mathrm{mp}>250{ }^{\circ} \mathrm{C}$; IR $\left(v_{\max }, \mathrm{cm}^{-1}\right): 3429,3179,1624,1544,1375,1348,1164,1082,951$, 851, 790. ${ }^{1} \mathrm{H}$ NMR (DMSO-d $): \delta_{\mathrm{H}} 2.49(3 \mathrm{H}, \mathrm{s}), 7.25(1 \mathrm{H}, \mathrm{s}), 7.41(1 \mathrm{H}, \mathrm{d}, J 9 \mathrm{~Hz}), 7.42(2 \mathrm{H}, \mathrm{d}, J$ $8 \mathrm{~Hz}), 7.59(1 \mathrm{H}, \mathrm{d}, J 9 \mathrm{~Hz}), 7.91(2 \mathrm{H}, \mathrm{d}, J 8 \mathrm{~Hz}), 8.30(1 \mathrm{H}, \mathrm{s}), 11.83(1 \mathrm{H}, \mathrm{s}), 13.09(1 \mathrm{H}, \mathrm{s}) .{ }^{13} \mathrm{C}$ NMR (DMSO- $\left.d_{6}\right): \delta_{\mathrm{C}} 21.2\left(\mathrm{PhCH}_{3}\right), 97.9,105.0,112.7,125.0,129.9,131.8$ (all $\left.\mathrm{CH}_{\text {arom }}\right), 115.7$, 119.4, 130.2, 131.6, 136.7, 136.8, 137.0 (all $\mathrm{C}_{\text {arom}}$ ). MS (ESI) calcd for $\mathrm{C}_{16} \mathrm{H}_{14} \mathrm{~N}_{3}(\mathrm{M}+\mathrm{H})$ 248.1187; found: 248.1107. Anal. Calcd for $\mathrm{C}_{16} \mathrm{H}_{13} \mathrm{~N}_{3}$ : C, 77.73; H, 5.26; N, 17.01\%. Found: C, $77.77 ; \mathrm{H}, 5.28 ; \mathrm{N}, 17.04 \%$.

7-(4-Methoxyphenyl)-3,6-dihydropyrrolo[3,2-e]indazole (13h). Colorless solid, yield 86\%, $0.226 \mathrm{~g}, \mathrm{mp}>250^{\circ} \mathrm{C}$; IR $\left(v_{\max }, \mathrm{cm}^{-1}\right): 3425,3163,1607,1546,1495,1253,1180,1025,828$, 785. ${ }^{1} \mathrm{H}$ NMR (DMSO- $\left.d_{6}\right): \delta_{\mathrm{H}} 3.77(3 \mathrm{H}, \mathrm{s}), 7.00(2 \mathrm{H}, \mathrm{d}, J 8 \mathrm{~Hz}), 7.01(1 \mathrm{H}, \mathrm{s}), 7.20(1 \mathrm{H}, \mathrm{d}, J 9$ $\mathrm{Hz}), 7.42(1 \mathrm{H}, \mathrm{d}, J 9 \mathrm{~Hz}), 7.76(2 \mathrm{H}, \mathrm{d}, J 8 \mathrm{~Hz}), 8.14(1 \mathrm{H}, \mathrm{s}), 11.62(1 \mathrm{H}, \mathrm{s}), 12.92(1 \mathrm{H}, \mathrm{s}) .{ }^{13} \mathrm{C}$ NMR (DMSO- $\left.d_{6}\right): \delta_{\mathrm{C}} 55.6\left(\mathrm{OCH}_{3}\right), 97.3,104.6,112.8,114.8,126.4,131.9$ (all $\left.\mathrm{CH}_{\text {arom }}\right), 115.6$, $119.5,125.6,131.4,136.8,137.0,158.9$ (all $\mathrm{C}_{\text {arom}}$ ). MS (ESI) calcd for $\mathrm{C}_{16} \mathrm{H}_{14} \mathrm{~N}_{3} \mathrm{O}(\mathrm{M}+\mathrm{H})$ 264.1137; found: 264.1100. Anal. Calcd for $\mathrm{C}_{16} \mathrm{H}_{13} \mathrm{~N}_{3} \mathrm{O}$ : C, 73.00; H, 4.94; N, 15.97\%. Found: C, $73.05 ; \mathrm{H}, 4.96 ; \mathrm{N}, 15.99 \%$.

\section{Acknowledgements}

Professor M. Chakrabarty and Dr. Sujay K. Barik thank the CSIR, Govt. of India for the grant (to MC) of an Emeritus Scientist Project (Sanction No. 21(824)/10/EMR-II, dated 23.12.2010) and a Research Associateship (to SKB). Professor Gandhi K. Kar and Mrs. Maumita Rakshit thank the Vice-Chancellor, Presidency University for laboratory facilities and the CSIR, Govt. of India for the award of a CSIR-NET Fellowship (to MR). All the authors are also thankful to Mr. B. Majumdar and Mr. S.R. Maji, both of the Bose Institute, for recording the NMR and GC-EI-MS spectra, respectively.

\section{References}

1. Horton, D. A.; Bourne, G. T.; Smyth, M. L. Chem. Rev. 2003, 103, 893. http://dx.doi.org/10.1021/cr020033s

2. Brancale A.; Silvestri, R. Med. Res. Rev. 2007, 27, 209. http://dx.doi.org/10.1002/med.20080

3. MDL Drug Data Report; MDL Information Systems Inc.: San Leandro, CA, USA. 
4. Cerecetto, H.; Gerpe, A.; González, M.; Arán, V. J.; de Ocáriz, C. O. Mini-Rev. Med. Chem. 2005, 5, 869. http://dx.doi.org/10.2174/138955705774329564

5. Runti, C; Baioocchi, L. Int. J. Tissue React. 1985, 7, 175.

6. Cioli, V; Corradino, C; Barcellona, P. S. Int. J. Tissue React. 1985, 7, 205.

7. Rakshit, M.; Kundu, T.; Kar, G. K.; Chakrabarty, M. Monatsh. Chem. 2013, 144, 717. http://dx.doi.org/10.1007/s00706-012-0859-5

8. Chakrabarty, M.; Mukherji, A. J. Heterocycl. Chem. 2012, 49, 1443. http://dx.doi.org/10.1002/jhet.992

9. Chakrabarty, M.; Mukherji, A.; Karmakar, S.; Mukherjee, R.; Nagai, K.; Geronikaki A.; Eleni, P. Arkivoc 2010, (xi), 265. http://dx.doi.org/10.3998/ark.5550190.0011.b22

10. Saik, F. H.; Kar, G. K. Beilstein J. Org. Chem. 2009, 5, 1.

11. Chakrabarty, M.; Kundu, T.; Arima S.; Harigaya, Y. Tetrahedron 2008, 64, 6711. http://dx.doi.org/10.1016/j.tet.2008.05.009

12. Chakrabarty, M.; Mukherji, A.; Mukherjee, R.; Arima, S.; Harigaya, Y. Tetrahedron Lett. 2007, 48, 5239. http://dx.doi.org/10.1016/j.tetlet.2007.05.144

13. Chakrabarty, M.; Kundu, T.; Arima S.; Harigaya, Y. Tetrahedron Lett. 2005, 46, 2865. http://dx.doi.org/10.1016/j.tetlet.2005.02.125

14. Chakrabarty, M.; Basak, R.; Harigaya Y.; Takayanagi, H. Tetrahedron 2005, 61, 1793 and references cited therein.

http://dx.doi.org/10.1016/j.tet.2004.12.022

15. Crapcho, P.; Menta, E.; Oliva, A.; Domenico, R. Di.; Fiocchi, L.; Maresch, M. E.; Gallagher, C. E.; Hacker, M. P.; Beggiolin, G.; Giuliani, F. C.; Perzoni, G.; Spinelli, S. J. Med. Chem. 1998, 41, 5429.

16. Schmidt, A.; Snovydovych, B.; Hemmen, S. Eur. J. Org. Chem. 2008, 25, 4313.

17. Lyubchanskaya, V. M.; Alekseeva, L. M.; Savina, S. A.; Granik, V. G. Chem. Heterocycl. Comp. 2000, 36, 1276.

http://dx.doi.org/10.1023/A:1017515300244

18. McEvoy, F. J.; Smith J. M. Jr.; Allen, D. S. Jr. US Pat. 3404157 (1968).

19. Maksimov, N. Ya.; Chetverikov, V. P.; Kost, A. N. SU Pat. 685664 (1979).

20. Singh, R.; Bhagavateeswaran, H.; Jain, P. C.; Anand, N. Indian J. Chem. 1982, 21 B, 853.

21. Sequeria, S.; Seshadri, S. Indian J. Chem. 1987, $26 B, 436$.

22. Borza, I.; Bozó, É.; Barta-Szalai, G.; Kiss, C.; Tárkányi, G.; Demeter, A.; Gáti, T.; Háda, V.; Kolok, S.; Gere, A.; Fodor, L.; Nagy, J.; Galgóczy, K.; Magdó, I.; Ágai, B.; Fetter, J.; Bertha, F.; Keserü, G. M.; Horváth, C.; Farkas, S.; Greiner I.; Domány, G. J. Med. Chem. 2007, 50, 901.

http://dx.doi.org/10.1021/jm060420k 
23. Spyridonidou, K.; Fousteris, M.; Antonia, M.; Chatzianastasiou, A.; Papapetropoulos, A.; Nikolaropoulos, S. Bioorg. Med. Chem. Lett. 2009, 19, 4810. http://dx.doi.org/10.1016/j.bmcl.2009.06.047

24. Bronner, S. M.; Goetz, A. E.; Garg, N. K. Synlett 2011, 2599.

25. Bronner, S. M.; Goetz, A. E.; Garg, N. K. J. Am. Chem. Soc. 2011, 133, 3832. http://dx.doi.org/10.1021/ja200437g

26. Gavara, L.; Anizon, F.; Moreau, P. Tetrahedron 2011, 67, 7330. http://dx.doi.org/10.1016/j.tet.2011.07.029

27. Gavara, L.; Suchaud, V.; Nauton, L.; Théry, V.; Anizon, F.; Moreau, P. Bioorg. Med. Chem. Lett. 2013, 23, 2298. http://dx.doi.org/10.1016/j.bmcl.2013.02.074

28. Massa, S; Stefancich, G; Artico, M.; Corelli, F.; Silvestri, R. Il Farmaco 1985, 42, 567.

29. Silva, V. L. M.; Silva, A. M. S.; Pinto, D. C. G. A.; Cavaleiro, J. A. S. Synlett 2006, 1369. http://dx.doi.org/10.1055/s-2006-939715

30. Silva, V. L. M.; Silva, A. M. S.; Pinto, D. C. G. A.; Elguero, J.; Cavaleiro, J. A. S. Eur. J. Org. Chem. 2009, 74, 4468. http://dx.doi.org/10.1002/ejoc.200900513

31. Starosotnikov, A. M.; Khakimo, D. V.; Bastrakov, M. A.; Pechenkin, S. Y.; Shevelev, S. A.; Pivina, T. S. Chem. Heterocycl. Comp. 2011, 47, 215. http://dx.doi.org/10.1007/s10593-011-0743-y

32. Starosotonikov, A. M.; Bastrakov, M. A.; Pechenkin, S. Y.; Leontieva, M. A.; Kachala, V. V.; Shevelev, S. A. J. Heterocycl. Chem. 2011, 48, 824. http://dx.doi.org/10.1002/jhet.599

33. Wahyuningsih, T. D.; Pechalek, K.; Kumar, N.; Black, D. StC. Tetrahedron 2006, 62, 6343. http://dx.doi.org/10.1016/j.tet.2006.04.041

34. Sundberg, R. J. Pyrroles and Their Benzoderivatives: Synthesis and Applications. In Comprehensive Heterocyclic Chemistry; Katritzky, A. R.; Rees, C. W., Eds.; Pergamon: Oxford, 1984, Vol. 4, 313. http://dx.doi.org/10.1016/B978-008096519-2.00056-4

35. Joule, J. A. Indole and Its Derivatives. In Science of Synthesis: Houben-Weyl Methods of Molecular Transformations: Thomas, E. J. Ed.; Georg Thieme Verlag: Stuttgart, 2000; Category 2, Vol. 10, Chapter 10.13.

36. Joule, J. A. In Comprehensive Heterocyclic Chemistry III; Katritzky, A. R., Rees, C. W., Scriven, E. F. V., Taylor, R. J. K., Eds.; Elsevier Science: United States, 2008; Vol. 4, Chapter 4.0.1.

37. Li, J. J.; Gribble, G. W.; Palladium in Heterocyclic Chemistry; Pergamon: Oxford, UK, 2000, Chapter 3, 73.

38. Cacchi, S.; Fabrizi, G. Chem. Rev. 2005, 105, 2873.

39. Battistuzzi, G.; Cacchi, S.; Fabrizi, G. Eur. J. Org. Chem. 2002, 2671. http://dx.doi.org/10.1002/1099-0690(200208)2002:16<2671::AID-EJOC2671>3.0.CO;2-X 
40. Arcadi, A.; Cacchi, S.; Carnicelli, V.; Marinelli, F. Tetrahedron 1994, 50, 437. http://dx.doi.org/10.1016/S0040-4020(01)80766-3

41. Cacchi, S.; Fabrizi, G.; Parisi, L. M. Synthesis 2004, 1889. http://dx.doi.org/10.1021/jm020077t

42. Flynn, B. L.; Hammel, E.; Jung, M. K. J. Med. Chem. 2002, 45, 2670. http://dx.doi.org/10.1021/jm020077t

43. Sonogashira, K.; Tohda, Y.; Hagihara, N. Tetrahedron Lett. 1975, $50,4467$. http://dx.doi.org/10.1016/S0040-4039(00)91094-3

44. Sonogashira, K. In Handbook of Organopalladium Chemistry for Organic Synthesis; Negishi, E. Ed.; John Wiley \& Sons: New York, 2002, Vol. 1, 493. http://dx.doi.org/10.1002/0471212466.ch22

45. Chinchilla, R.; Nájera, C. Chem. Rev. 2007, 107, 874. http://dx.doi.org/10.1021/cr050992x

46. Chinchilla, R.; Nájera, C. Chem. Soc. Rev. 2011, 40, 5084. http://dx.doi.org/10.1039/c1cs15071e

47. Evano, G.; Blanchard, N.; Toumi, M. Chem. Rev. 2008, 108, 3054. http://dx.doi.org/10.1021/cr8002505

48. Nishihara, Y.; Inoue, E.; Ogawa, D. ; Okada, Y.; Noyori, S.; Takagi, K. Tetrahedron Lett. 2009, 50, 4643.

http://dx.doi.org/10.1016/j.tetlet.2009.05.112

49. Spań, V.; Montalbano, A.; Carbone, A.; Parrino, B.; Diana, P.; Cirrincione, G.; Barraja, P. Tetrahedron 2013, 69, 9839.

http://dx.doi.org/10.1016/j.tetlet.2009.05.112 